\title{
Philosophiques
}

\section{Les objets culturels}

\section{Yann Nicolas}

Volume 26, numéro 2, automne 1999

La critique de la raison en Europe centrale

URI : https://id.erudit.org/iderudit/004962ar

DOI : https://doi.org/10.7202/004962ar

Aller au sommaire du numéro

\section{Éditeur(s)}

Société de philosophie du Québec

\section{ISSN}

0316-2923 (imprimé)

1492-1391 (numérique)

Découvrir la revue

\section{Citer cet article}

Nicolas, Y. (1999). Les objets culturels. Philosophiques, 26(2), 301-314.

https://doi.org/10.7202/004962ar

\section{Résumé de l'article}

Le dernier étage de l' Aufbau de Carnap est occupé par le type des objets culturels (État, coutume, groupe social). L'examen des remarques fugitives de Carnap et la formulation des embarras ou des lacunes qu'elles manifestent montrent notamment, de l'intérieur, les faiblesses du cadre extensionnaliste en ce qui concerne la compréhension des objets culturels. La mention comparative de certains essais phénoménologiques d'ontologie des objets culturels (Husserl, E. Stein) suggère d'autres voies (ou bien rappelle l'extensionnaliste à ses devoirs).
Ce document est protégé par la loi sur le droit d'auteur. L'utilisation des services d'Érudit (y compris la reproduction) est assujettie à sa politique d'utilisation que vous pouvez consulter en ligne.

https://apropos.erudit.org/fr/usagers/politique-dutilisation/ 


\title{
Les objets culturels
}

\author{
YANN NICOLAS \\ ynicolas@hotmail.com
}

\begin{abstract}
RÉSUMÉ. - Le dernier étage de l'Aufbau de Carnap est occupé par le type des objets culturels (État, coutume, groupe social). L'examen des remarques fugitives de Carnap et la formulation des embarras ou des lacunes qu'elles manifestent montrent notamment, de l'intérieur, les faiblesses du cadre extensionnaliste en ce qui concerne la compréhension des objets culturels. La mention comparative de certains essais phénoménologiques d'ontologie des objets culturels (Husserl, E. Stein) suggère d'autres voies (ou bien rappelle l'extensionnaliste à ses devoirs).
\end{abstract}

\begin{abstract}
The last floor of the Carnap's Aufbau by is inhabited by the type of cultural objects (State, custom, social group). The examination of Carnap's brief remarks and the formulation of the embarrassments or the lacunas they exhibit show, from within, the weaknesses of the extensionalist framework for the comprehension of cultural objects. The comparative mention of phenomenological attempts at an ontology of cultural objects (Husserl, E. Stein) suggests other ways of handling them (or reminds the extensionalist of his duties).
\end{abstract}

Le titre de cet article ex cède son contenu, car il ne consistera qu'en quelques remarques sur les efforts marginaux des principaux représentants de deux courants majeurs de la philoso phie autrichienne, à savoir Carnap et $\mathrm{H}$ usserl. Ces efforts marginaux et programmatiques, ce sont des essais d'extension de leurs projets philosophiques respectifs, empirisme logique et phénoménologie, à un nouveau domaine d'objets dont l'essor des sciences de l'homme (ou « sciences de l'esprit ») a montré l'importance, et les suggestions de Dilthey la nécessité d'un traitement philosophique. $\mathrm{N}$ i chez $\mathrm{C}$ arnap ni chez $\mathrm{H}$ usserl, ces essais n'ont abouti à quelque chose; chez nos deux auteurs, ces efforts donnent parfois l'impression de servir de gage de bonne conscience à un souci d'exhaustivité du projet philosophique. D'un côté, la marginalité des analyses des objets culturels risque de restreindre leur capacité d'innovation, mais d'un autre côté, cette marginalité exerce une contrainte féconde en imposant qu'on aborde les objets culturels par une attitude inédite puisque forgée pour d'autres fins et d'autres domaines d'objets. D'où la pertinence d'une lecture spécifique de ces analyses qui ne sont pas déductibles a priori des principes généraux des grands programmes dont elles occupent les « strapontins ». Je suggérerai en conclusion que ces analyses sont, dans une grande mesure, indépendantes des positions de leur programme-hôte à l'égard des « grandes» questions métaphysiques.

Documentation et manifestation

Avant de commenter les notions de document et de manifestation, je ferai quelques rappels au sujet de l'A ufbau. 
On sait que la tâche de Carnap dans l'A ufbau est de discuter de la possibilité et des modalités d'un système constructionnel exhaustif et d'en proposer seulement l'esquisse d'une version. Un système constructionnel se présente comme une chaîne de définitions qui permettent de réduire les objets de la science et du sens commun à une base d'objets restreinte. Un système constructionnel est aux objets (ou aux concepts) ce qu'uneaxiomatique est aux propositions. O n sait aussi que, pour Carnap, le critère de correction des définitions constructionnelles est l'identité extensionnelle : I'analyse ne s'intéresse qu'aux extensions des propriétés des objets à réduire; un type d'objets sera réductible à un autre si on peut traduire tout ce qu'on dit du premier par ce qu'on dit du second, et pourvu que toutes les traductions pré servent la valeur de vérité des phrases. Pour rendre effectif le critère extensionnaliste, Carnap préconise de le compléter par un critère factuel : plus précisément, dans le but de s'assurer de l'identité extensionnelle entre deux objets ou concepts, on fait appel aux sciences empiriques pour nous fournir des faits qui soient les conditions nécessaires et suffisantes des faits représentatifs de l'objet à réduire. $M$ ais ce critère factuel ne suffit pas à départager la candidature d'un système à base physique et celle d'un système à base psychique. Pour ce faire, il faut introduire un nouveau critère qui exige que les conditions factuelles soient aussi des indicateurs de l'objet à réduire. Ce critère de priorité épistémique privilégie un système phénoménaliste, puisquele fait primitif qu'est la perception fait que, nécessairement, tout objet physique est connu après et par un objet psychique, en l'occurrence, un acte perceptif.

L'A ufbau s'intéresse principalement à trois types d'objets : les objets psychiques, les objets physiques et les objets culturels. Pour que les objets culturels soient réductibles aux autres types, il suffit de trouver une relation qui, en préservant les exigences de coextensivité et de priorité épistémique, lie les objets culturels soit aux objets physiques, soit aux objets psychiques. $D$ ans le premier cas, cette relation sera la relation de documentation et reliera tous les objets culturels à leurs indicateurs physiques, les documents. Dans le second cas, cette relation sera la relation de manifestation et reliera tous les objets culturels à leurs indicateurs psychiques, leurs manifestations. Carnap affirme que ces deux relations existent. II faut, semble-t-il, en conclure qu'à ce niveau (mais pas au niveau du système entier, on l'a vul) on peut indifféremment réduire le culturel au physique ou au psychique. $M$ ais ce serait négliger certains flottements carnapiens à propos de la notion de document.

D'abord, remarquons que le terme même de « document » et les exemples qu'en donne Carnap créent une certaine ambiguïté. En effet, en histoire 2 ou en archéologie, on entend par « documents » des vestiges physiques por-

1. Puisque, si le psychique et le physique sont tous deux épistémiquement premiers par rapport au culturel, ils ne peuvent l'être l'un par rapport à l'autre ; I'ordre de la connaissance étant symétrique, I'un doit s'effacer.

2. Dans ces passages, Carnap semble penser à l'histoire plus qu'à d'autres sciences humaines. Ce privilège n'est pas exceptionnel dans les écrits de cette période sur le problème 
teurs de sens dont il faut retrouver la signification (pour les images, les écrits) ou la fonction (pour les outils, les bâtiments); il ne s'agit donc pas de purs objets physiques au sens où toutes leurs propriétés seraient physiques. De plus, si on peut réduire un objet culturel à ses seuls documents compris comme l'ensemble de ses traces physiques (y compris les comportements des individus), alors il faut accepter la thèse béhavioriste. Or, Carnap semble hésitant à ce sujet ${ }^{3}$.

L'ambiguïté du terme « document » devient patente au $\S 55$, puisque Carnap y précise la condition pour qu'un objet physique « devienne un document » : il faut un « acte de création ou de transformation de la part d'un ou plusieurs individus ». Selon Carnap, ce n'est que par ces actes psychiques que «l'objet culturel » prend vie. II est clair que la distinction entre les manifestations et les document(ation)s ne tient plus : les documents ne sont pas des objets physiques stricto sensu, mais seulement en tant qu'ils sont animés par les actes psychiques d'un membre d'une culture (voire d'un historien ${ }^{4}$; ces actes psychiques sont autant de manifestations de notre objet culturel. En ce sens, contrairement à ce que disent certains passages de $\mathrm{Car}$ nap, les documents sont un mélange de physique et de psychique. Cette mixité appelle deux commentaires d'ordres très différents.

Le premier commentaire est une remarque critique: si les documents ne peuvent être des objets physiques comme les autres, pourquoi Carnap ne conclut-il pas du constat de cette mixité plus ou moins stable à la reconnaissance d'une nouvelle espèce d'objets culturels, précisément ces documents, objets mixtes en tant qu'ils posséderaient des caractères physiques et des caractères symboliques, ou « prédicats de signification », comme H usserl les appelle? Pourquoi ne conçoit-il les documents que comme les produits d'une projection psychologique sur des objets physiques sans leur accorder un statut officiel? H usserl ${ }^{5}$, lui, inclut parmi les objets culturels les temples, les cuillers, les textes, ces objets qui, outre leurs propriétés physiques, possèdent, en tant qu'ils ont une signification ou une fonction, une intentionnalité seconde, dérivée (c'est pourquoi ce ne sont pas des objets psychiques). II les qualifie d' « objets investis d'esprit ». M ais l'exemple de H usserl n'est certes pas une raison suffisante pour cela.

Si notre question n'est pas totalement étrangère au texte de Carnap, c'est que précisément, au $\S 133$, il propose d'intégrer certaines projections psychologiques visant un objet de la perception à cet objet lui-même en tant

général des « sciences de l'esprit » et il témoigne souvent d'une certaine conception des rapports entre sciences de la nature (comme sciences du général) et sciences de l'esprit (comme sciences du particulier porteur de valeur) ; c'est le cas chez Rickert malgré ses protestations, mais il n'y a aucune raison de penser que ce le soit chez Carnap.

3. Carnap, 1928, § 24.

4. M ais ce deuxième cas pose d'autres problèmes: l'étude de l'historien ne fait pas renaître son objet.

5. H usserl, 1982. 
que propriétés réelles de cet objet. Par exemple, l'assignation de couleurs aux points du monde semble assez naturelle. D'autres assignations sont plus problématiques ; il semble moins naturel $d$ ' « assigner des qualités d'émotions ou de volitions comme propriétés des choses dans le monde extérieur ». Selon Carnap,

ces assignations sont complètement justifiées, car, exactement de la même manière que nous pouvons qualifier le sucre de « doux » du fait qu'il produit une sensation de goût d'une qualitéappropriée, une mélodie peut être qualifiée de « gaie », une lettre de « douloureuse » et un acte de « scandaleux », du fait que ces objets produisent les émotions appropriées.

Le raisonnement peut être étendu aux propriétés volitives, même s'il semble moins aisé de dire d'une pomme qu'elle est « demandeuse (ou solliciteuse) de croquage ». M ais ce n'est pas le caractère plus ou moins naturel de ces formulations qui décide si une assignation d'une propriété psychique à un objet physique est autorisée ou non ; on pourrait même fairel'hypothèse que c'est l'inverse : si une assignation est adoptée, elle finira bien par sembler naturelle ${ }^{6}$. Le critère ordinaire de distinction entre les assignations autorisées et les autres n'est pas non plus, selon Carnap, leur invariance temporelle, puisque les assignations de température ou olfactives sont monnaie courante, alors que les assignations émotionnelles et volitives semblent indésirables; or les émotions et les volitions semblent plus stables que les températures ou les odeurs, du moins tant que l'on ne considère que le flux de l'expérience d'un sujet unique, avant que soient construits les autres sujets et le monde intersubjectif. Précisément, dès que plusieurs sujets différents sont disponibles, on remarque qu'ils s'accordent mal entre eux sur les assignations émotionnelles et volitives. Un moyen efficace d'éviter des assignations contradictoires est tout bonnement de les interdire ou de les marginaliser. $C^{\prime}$ 'est ce que l'on fait ordinairement.

N ous pouvons maintenant revenir à nos candidats au titre d'objets physiques culturels, à nos objets symboliques ou fonctionnels ou encore institutionnels, à nos textes, nos images, nos outils et nos armes (au sens technique ou juridique). Si les droits de passage à acquitter pour passer du statut de cibles des projections psychologiques au statut de supports, de porteurs de propriétés (intrinsèques et) non physiques sont une certaine invariance et surtout une fiabilité intersubjective, nos candidats semblent être des candidats tout à fait recevables et même plutôt doués. En effet, les assignations de signification ou de fonction paraissent plus stables que les assignations de températures et plus consensuelles ${ }^{7}$ que les assignations d'émotions. C'est pourquoi le discours de l'historien de l'art est plus invariant que celui du présentateur météo et mieux partagé que celui du critique d'art. II n'y a donc

6. Comme une métaphore devient catachrèse.

7. Cette fiabilité intersubjective des symboles ou des outils n'est pas étonnante puisqu'elle est sans doute constitutive de leur caractère conventionnel, que ce caractère soit établi via les actes de langage searliens (The Construction of Social R eality) ou par une émergence plus spontanée, 
plus d'obstacle à considérer le texte, le tableau ou l'outil comme des objets culturels à part entière, et non plus comme des objets strictement physiques qui serviraient régulièrement de cible à des projections psychologiques particulières. Le fait d'avoir telle signification, de représenter tel personnage ou de remplir telle fonction, est intégré à un objet qui entretient certaines relations avec des objets physiques (le papier, la toile, le tube métallique) et des objets psychiques (jugements, croyances, dispositions). L'analyse se chargera de mettre en évidence ces relations et de réduire ces objets culturels primaires. L'A ufbau autorise une telle conception des « documents », y conduit même, mais nel'adopte jamais. Certes, elle ne change rien à la réduction ellemême, mais elle est compatible avec nos intuitions ordinaires et certaines remarques de Carnap.

Le deuxième commentaire concerne, d'une certaine manière, le rapport entre l'ordre philosophique de la construction et l'ordre scientifique de l'explication. On sait que Carnap opte en 1928 pour une base phénoménaliste. Cette décision implique-t-il un choix en faveur d'un programme scientifique particulier? S'agissant des sciences sociales, le phénoménalisme de la construction interdit-il une méthode matérialiste, béhavioriste par exemple? Dans Sociologie empirique (1931) de N eurath, le physicalisme impose un marxisme revu et corrigé par un béhaviorisme.

II semble que la réponse à cette question ne dépende pas seulement de la nature de la base du système, mais de l'ensemble du profil des réductions. Si le système de Carnap n'est pas compatible avec le béhaviorisme, ce n'est pas à cause du choix de sa base, mais parce que, comme nous l'avons vu en parlant des documents, il déclare impossible la construction des objets culturels à partir des seuls objets physiques. Pour ce faire, il faut que les objets physiques soient animés par des actes psychologiques. $0 \mathrm{n}$ voit que les objets psychiques ne servent pas seulement à construire les objets physiques, mais aussi les objets culturels. O n ne peut donc pas isoler dans la structure du système entier un système local physicaliste où les objets culturels seraient bel et bien réduits. C'est l'impossibilité d'un tel sous-système, et seulement elle, qui rend, par exemple, incompatibles le système phénoménaliste de Carnap et le béhaviorisme.

\section{Mise en perspective de l'Aufbau}

A près avoir présenté et discuté les notions de manifestation et de documentation, qui suffisent, sel on Carnap, à rendre possible une réduction systématique du langage de la culture, je voudrais donner une idée de ce que signifierait l'achèvement d'une telle réduction. Je le ferai en situant le projet

dans le « common knowledge» de Lewis (Convention). Cependant, comme le fait remarquer Searle, les fonctions des objets physiques fonctionnels sont plus ou moins enracinées dans les propriétés strictement physiques de leur support (cf. la différence entre de l'argent et une chaise). 
de Carnap dans le contexte plus ou moins fictif d'une concurrence avec d'autres essais philosophiques pour comprendre le monde et les sciences de la culture.

Carnap reconnaît que l'introduction des « sciences de l'esprit » dans le débat philosophique, voire ontologique, doit être attribuée à Dilthey. En A utriche, comme en Allemagne et en France, cette introduction, qui est parfois vécue comme une intrusion, ouvre et nourrit les querelles sur l'unité de la science et les analogies que l'on peut faire entre les sciences de l'esprit et les sciences de la nature. Faut-il voir cette nouveauté comme l'espoir d'un précarré humaniste échappant à la législation de la mécanique (Dilthey, R ickert)? Faut-il y voir la menace d'une imposture visant à s'approprier à peu de frais le prestige, à défaut des pouvoirs et des acquis, d'une science qui a fait ses preuves (Poincaré soupçonnant les virtuosités de Walras)? O u ne doit-on interpréter cette introduction que comme l'extension tranquille d'une «physique » qui enfin conquiert un nouveau domaine, le domaine social (Comte, Durkheim)? O n ne peut négliger des positions plus nuancées, telles que celle du fondateur de l'économie autrichienne $C$ arl $M$ enger ou celle de $M$ ax Weber.

La prise en charge de cette querelle institutionnelle, méthodologique, épistémologique et ontologique par certains des membres du Cercle de Vienne est toujours médiatisée et marginalisée par deux préoccupations plus centrales que partagent tous les empiristes logiques : Ie défi de l'unification de la science et le souci normatif d'élimination des discours métaphysiques. II faut dire sans délai que ces deux préoccupations ne sont pas indépendantes l'une de l'autre : I'intégration est le revers de l'exclusion.

Pour Carnap comme pour $\mathrm{N}$ eurath, le problème que pose la seule existence des sciences de la culture ne peut échapper à l'alternative suivante : ou bien elles s'intègrent au corps des sciences traditionnelles qu'on s'évertue à constituer à force de paraphrases, ou bien elles se situent hors du régime linguistique de la science authentifiée et se perdent dans la métaphysique. La seule nuance qu'admet cette al ternative est d'autoriser qu'on trace cette ligne de démarcation à l'intérieur même de l'ensemble des émissions des sciences de la culture et non seulement à ses frontières extérieures : c'est à ce tri interne que se consacre $\mathrm{N}$ eurath pour ne retenir que ce qui paraît compatible avec son hétérodoxe marxisme béhavioriste. Carnap sera plus «tolérant » (pour reprendre l'expression qu'il adoptera en 1934). Toujours est-il qu'il n'est certainement plus question d'aménager dans l'édifice de la Science Unifiée une aile autonome qui abriterait les sciences de la culture, pas plus que ne serait compatible avec l'optimisme positiviste le rejet en bloc de cette nouvelle source de connaissances; toutes les sciences seront donc logées à la même enseigne.

$M$ ais la description de ce programme d'intégration/exclusion ne rend pas justice au traitement des objets culturels par Carnap en 1928, bien qu'il soit plus fidèle à son traitement encore plus allusif de 1934 (The U nity of 
Science). En effet, le simple vocabulaire spatial de l'unification, de l'exclusion ou del'inclusion ne peut rendre les nuances et les complexités de la solution qu'autorise la forme des systèmes constructionnels. Ce n'est pas la moindre des vertus de la construction logique que de souligner la plurivocité de la question de l'unité des sciences et la nécessité de réponses circonstanciées, ce que ni les quer elles allemandes ni le slogan viennois n'accomplissent. La sophistication de l'architecture d'un système constructionnel permet de distinguer différents aspects de la question, distinction qui conduit à une réponse complexe qu'une traduction impatiente dans les termes plurivoques et échauffés par les enjeux académiques ne peut rendre fidèlement.

L'ensemble de la construction rapportée aux objets culturels, c'est-àdire aux objets des sciences del'esprit mais aussi de ce qu'on pourrait appeler le « sens commun culturel », présente des prises de position originales et circonstanciées par rapport à la plupart des autres prises de position philosophiques qui essayent de penser les sciences de l'esprit.

Carnap tient à assurer l'autonomie des objets culturels (comme le font Dilthey ou Edith Stein) tout en défendant l'unité de la science (commele fait $\mathrm{N}$ eurath). $\mathrm{M}$ ais il faut reconnaître qu'il ne parvient pas à donner une définition adéquate de cette autonomie.

De manière corrélative, il refuse de tenir pour irréductibles les objets du sens commun culturel (contre Stein) mais leur accorde l'existence (contre $\mathrm{H}$ ayek et $\mathrm{N}$ eurath) ; ici, il est proche de certains textes de Weber. Plus précisément, non seulement il essaye de sauver l'existence des objets culturels malgré la réduction, mais il veut la sauver par la réduction : la réduction de tous les types d'objets au seul domaine des objets autopsychologiques n'implique nullement, dans l'idée de Carnap, de refuser aux objets réduits l'existence. Un tel interdit ne pourrait se faire, en effet, qu'au profit d'un sens extraordinaire de la notion d'existence, étranger au discours naïf mais légitime de l'existence quotidienne, juridique ou scientifique. Ce sens extraordinaire de l'existence serait un statut métaphysique de l'existence qui n'a pas sa place dans un système constructionnel. Par contre, l'idée naïve d'existence est, à cet égard, inoffensive, même si sa naïveté l'expose à une interprétation métaphysique clandestine, d'où la préférence de $\mathrm{C}$ arnap pour le « langage constructionnel » par rapport au « langage des états de chose », pour le « mode formel » par rapport au « mode matériel ». $M$ algré cette réserve, Carnap ne voit pas de mal, bien au contraire, à ce que se reflète dans le système lui-même la prétention légitime des objets construits à l'existence. Ainsi, au § 171, il écrit qu'un objet culturel « est appelé réel si ses manifestations font partie des objets psychologiques réels ». L'existence des objets construits est donc fonction de l'existence des objets à partir desquels ils ont été construits.

Enfin, son antipsychologisme (contre Dilthey) ne lui interdit pas (encore) de souligner les relations étroites entre les objets culturels et les actes psychologiques (comme $\mathrm{M}$ enger, contre $\mathrm{N}$ eurath). 
Ces conciliations constituent autant d'indéterminations qui laissent au travail effectif d'investigation scientifique toute une gamme de méthodes et de langages. La position de Carnap est précieuse en ceci que son souci de neutralité et de tolérance lui interdit de franchir les limites qui séparent la philosophie de la méthodologie (si ce n'est l'incompatibilité de son système avec le béhaviorisme! $\mathrm{M}$ ais cette incompatibilité ne vaut que pour l'esquisse de système qu'il permet. II n'y a aucun interdit commun à tous les systèmes possibles). Cette prudence lui permet d'éviter de se payer le ridicule trop répandu et banalisé par lequel le philosophe, mystérieusement, miraculeusement mieux informéou plus pénétrant, écrit le cahier des charges quele sociologue ou l'historien sera censé respecter, en termes ontologiques et épistémologiques.

\section{Existence et dispositions}

Pour circonstancié et prudent qu'il soit, le programme de Carnap n'en est pas moins audacieux et même décapant. Cela ne va pas sans risques. $N$ ous allons nous attarder sur un de ces risques. Si je parle de risque, c'est bien pour souligner que toute critique éventuelle devra aller d'aval en amont, c'est-à-dire déplorer les conséquences incontrôlables et indésirables d'un choix, et non pas l'intention même qui préside à ce choix.

A u cours de ses remarques sur une construction possible des objets culturels, Carnap rencontre une difficulté que je crois représentative des risques de son parti pris extensionnaliste. Carnap ne fait que l'évoquer et ne semble pas la juger très dangereuse ; il l'enterre par une solution presque verbale qui est aussi bien une fin de non recevoir. N ous verrons pourtant qu'elle rejoint une difficulté qu'il reconnaîtra comme sérieuse quel ques années plus tard et qu'un cadre strictement extensionnel ne saurait lever. Cet écueil est le suivant.

$\mathrm{N}$ ous avons vu que la réduction de tous les types d'objets au seul domaine des objets autopsychologiques n'implique nullement, dans l'idée de Carnap, de refuser aux objets réduits l'existence. L'existence des objets construits est simplement dérivée de l'existence des objets à partir desquels ils sont construits. Tout cela paraît naturel et bien venu. $M$ ais $C$ arnap n'est pas sans s'apercevoir d'une conséquence indésirable de ce principe, indésirable eu égard à une intuition ordinaire, extra-systématique, qui n'est guère suspecte. Cette conséquence est la suivante : si, dès que ses manifestations psychologiques cessent d'exister, un objet culturel cesse d'exister, une coutume, par exemple, cessera d'exister quand aucun individu n'aura le comportement qui lui correspond, associé aux états psychiques qui lui correspondent. Ainsi, dans une société disciplinée de couche-tôt, par exemple un monastère, les coutumes qui l'habitent deviendraient inexistantes dès les lumières éteintes et renaîtraient à l'aube (à moins que ce ne soient de nouvelles coutumes, selon nos critères d'identité et de réidentification). Faudrait-il désigner alors un veilleur-denuit-conservateur-des-coutumes chargé d'interdire au péché ou à l'anarchie d'investir la communauté? Si l'on prend au sérieux le principe formulé par Carnap, il n'y a pas de raison de ne pas prendre au sérieux cette hypothèse. 
Il y a deux manières naturelles d'affronter cette difficulté. Carnap opte pour la seconde, sans en mesurer toutes les conséquences.

La première attitude, résignée, est implicitement adoptée par nos moines inquiets de leur fidélité à leurs règles : le principe de Carnap est adopté et complété par un second principe qui accorde une existence intermittente aux coutumes. II ne reste plus à la communautéqu'à faire en sorte que les périodes d'inexistence des coutumes soient minimales et tendanciellement nulles. L'idée de l'intermittence de l'existence n'est pas en soi contre-intuitive, mais, concernant notrecas, deux situations sont à distinguer, cequerien jusqu'ici ne permet de faire. II y a une bonne et une mauvaise intermittence des coutumes et des objets culturels en général : il semble naturel et désirable de qualifier d'intermittente une coutume qui s'éteint pendant mille ans puis réapparaît ; mais la situation est différente quand l'interruption ne dure qu'une nuit, ou pourquoi pas, quelques instants. C'est ce deuxième cas qui pose problème, alors que l'intermittence convient bien au premier. Et faire remarquer que la frontière est vague entre les deux situations n'autorise pas à dire qu'il n'y a pas de frontière. $O \mathrm{n}$ peut interpréter dans ce sens la solution seulement verbale que $C$ arnap évoque à son problème : dire, comme il le fait au $\S 24$, que pendant l'absence momentanée de ses manifestations, la coutume existebien mais de manière « latente », c'est indiquer que la solution par l'intermittence n'est pas toujours pertinente, et ne l'est pas dans le cas qui l'occupe.

Si la notion d'intermittence ne fournit pas une solution générale, la notion de « latence » n'en fournit pas de claire et distincte. N éanmoins, elle en suggère une que des passages de l'A ufbau explicitent sans s'y attarder et que la suite de l'histoire de la philosophie impose. En effet, l'idée de latence est bien celle d'une présence non actual isée, que l'idée de disposition netrahit guère. Or Carnap écrit sans s'y arrêter, en parlant de la coutume de saluer en soulevant son chapeau : "C Cette coutume n'existe pas seulement durant les moments où quelqu'un quelque part la manifeste, mais aussi durant les temps intermédiaires, aussi longtemps qu'il existe des personnes qui ont la disposition psychologique à réagir à certaines impressions en saluant quelqu'un en soulevant son chapeau. $»^{8}$

Le problème est que la notion de disposition crée autant de problèmes qu'elle en résout. Carnap en prendra conscience en 1936, dans « Testability and M eaning ", sans clore le dossier pour autant. II semble qu'une construction strictement extensionnaliste, qui identifie un objet culturel à ses manifestations, soit incapable de faire le partage entre la bonne et la mauvaise intermittence, entre une période sans manifestation mais avec disposition et une période sans manifestation ni disposition, entre l'état de latence et la non-existence. C'est pourquoi on peut voir, dans la nécessité d'une analyse des objets culturels par des dispositions envers certaines manifestations et non par des manifestations brutes, une des invitations possibles à abandonner un point de vue strictement extensionnaliste sur les objets culturels.

8. Carnap, 1928, § 24. 
On le sait, le défaut externe du recours à l'identité extensionnelle est de confondre des choses qu'on aimerait distinguer. C'est cette même avarice quant aux distinctions qui, du point de vue interne, du point de vue même de l'extensionnaliste, caractérise la difficulté du compagnonnage quand il s'agit pour Carnap de construire les (classes de) qualités à partir de la seule extension de la relation de base, la ressemblance mémorielle : dans certaines circonstances, l'extension d'une qualité accompagne toujours l'extension d'une autre qualité ; ce parasitage étant accidentel et non essentiel, il confond des choses dont la coïncidence est fortuite mais la distinction essentielle, et donc exigible de la construction. En plaidant les circonstances défavorables, $\mathrm{Car}$ nap espère les circonstances atténuantes.

$M$ ais le problème est que ces circonstances sont incontrôlables et peuvent se multiplier de manière dangereuse : il y a un risque irréductible de récidive. Carnap est conscient de ce risque quand il veut construire ces objets culturels supérieurs que sont les groupes sociaux. R efusant de confondre deux groupes partageant les mêmes membres, il renonce à construire les groupes comme des classes (c'est-à-dire comme des extensions de propriétés) et préfère les construire comme des extensions de relation, des listes de nuples. $C$ ette simple prudence témoigne de ce que $C$ arnap cherche à opérer un partage entre différentes identités extensionnelles (fiables et non fiables), ce que le recours aux extensions de relations, au-delà du seul recours aux classes, permet. $M$ ais cette méthode n'évacue pas le risque, même s'il se trouve allégé. En effet, le risque du compagnonnage demeure : deux relations constituant deux groupes différents peuvent être coextensives de manière contingente ; les deux groupes seraient identifiés, ce qui, dans certains cas, peut être totalement indésirable (par exemplesi l'on veut étudier les relations entre ces deux groupes).

$\mathrm{N}$ aturellement, le problème n'est pas qu'on se permette d'identifier des entités différentes sur la base d'une identité extensionnelle : cela voudrait dire que l'extensionnalisme est a priori illégitime, ce qui n'est pas notre propos. Le problème est plutôt que l'on distingue entre des identités extensionnelles fiables et non fiables, entre des circonstances normales et des circonstances défavorables, ce qui introduit une marge d'indétermination suspecte dans l'application de l'idée de reconstruction rationnelle extensionnaliste. On ne peut pas distinguer a priori quand on a affaire à une réduction légitime et quand on a affaire à une identification illégitime à cause d'un phénomène analogue au compagnonnage. En somme, tout se passe comme si Carnap voulait fonder ses réductions sur une nécessité plus forte que la simple coextensivité, afin d'écarter certaines coextensivités indésirables.

\section{$4 \quad$ Husserl et l'ontologie des objets culturels.}

Au moins cela signifie-t-il que le programme extensionnaliste n'est pas une machine aveugle ni exhaustive et que, dans certains cas, le constat d'une identité extensionnelle ne suffit pas à procéder à une identification systéma- 
tique. Le recours aux relations permet presque toujours de produire des extensions différentes pour des termes qui apparaissaient coextensifs mais qu'on se refusait à identifier. Cette censure provient de la nécessité des'accorder avec certaines intuitions extra-systématiques qui prononcent un interdit sur certaines réductions en triant parmi les identités extensionnelles le bon grain del'ivraie. $M$ ais le système extensionnaliste n'a rien à dire sur ces intuitions. II ne peut que prendre acte d'une différence intuitive entre les coïncidences trompeuses et les concomitances fiables. $0 \mathrm{n}$ peut pourtant penser que ces intuitions méritent une attention particulière de la part du philosophe.

Cette hypothèse est une manière d'aborder certains passages de $\mathrm{H}$ usserl, notamment un passage de Expérience et jugement, dans lequel il souligne que certaines identités extensionnelles sont fortuites, accidentelles, et non pas essentielles (pour parler en termes carnapiens) : « un objet idéal peut assurément, commela M adone de R aphaël, n'avoir en fait qu'une seule mondanéisation, et ne pas être, en fait, répétable dans une identité pleinement suffisante (celle du contenu idéal plein). $M$ ais par principe cet idéal est néanmoins répétable aussi bien que le Faust de G oethe. $»^{9}$

L'opposition entre « en fait » et « par principe » chez H usserl est parallèle à l'opposition entre « accidentel » et « essentiel » chez Carnap et à la distinction entreles « circonstances défavorables » et les circonstances ordinaires (celles qui autorisent une réduction légitime). Seulement, le strict extensionnalisme de Carnap lui interdit d'en dire plus sur ce genre de relations factuelles, alors que le cadre logico-ontologique de $\mathrm{H}$ usserl lui permet d'engager une analyse fragmentaire mais prometteuse des différentes espèces d'objets eu égard à cette distinction entre le fait et le principe. Cette analyse se penche notamment sur les rapports entre les objets culturels et leurs mondanéisations, c'est-à-dire, en termes carnapiens, les objets physiques qui les incarnent.

L'opposition husserlienne entre le fait et le principe doit être traduite dans les termes modaux de la contingence et de la nécessité. De même, la « possibilité de fait » peut être interprétée comme une possibilité naturelle, incluant ou non la possibilité technique, intermédiaire entre le simple fait actuel et la possibilité logique ou ontologique. En effet, les remarques de $\mathrm{H}$ usserl à propos des objets culturels relèvent d'une investigation ontologique attentive aux lois d'essence et non aux seules régularités ou coïncidences factuelles dont doit se contenter une perspective extensionnelle. Peter Simons et d'autres ont montré combien la notion de « dépendance » ou de « fondation » était centrale dans la philosophie de $\mathrm{H}$ usserl et comment on pouvait la reformuler dans les termes aiguisés et subtils de la logique contemporaine. On peut observer que, s'agissant de l'ontologie des objets culturels esquissée par H usserl, la notion de dépendance ontologique peut encore être d'un certain secours. 
Pour prendre un exemple, il me semble possible de reformuler la distinction husserlienne entre « idéalités liées » et « idéalités libres » dans les termes de la distinction soulignée par Simons entre dépendance ontologique rigide et dépendance ontologique non rigide. Selon $\mathrm{H}$ usserl, une œuvre d'art est une idéalité libre parce que son existence (au sens de l'existence dans le temps) dépend de l'existence d'un objet physique quelconque pourvu qu'il satisfasse à quelques conditions minimales. Au contraire, un État peut être conçu comme une idéalité liée, au sens où son existence dépend d'un territoire particulier, non d'un quelconque territoire. Avant Expérience et jugement, Edith Stein, dans son article sur l'État, s'était déjà interrogée sur les rapports entre l'État et son territoire. Qu'on accepte ou non de parler ici d'objets idéaux, ce qui est intéressant, c'est le parallèle entre le couple lié/ libre et le couple rigide/non rigide.

On sait que la notion de dépendance ontologique offre de grandes possibilités de diversification, diversification qui doit permettre de réfléchir la variété des relations qui existent entre les objets culturels et les objets physiques ou psychiques. L'introduction de paramètres temporels, notamment, sert cette diversification ${ }^{10}$.

Ainsi, ce qui chez $\mathrm{H}$ usserl motive la notion d'idéalité, c'est le fait que l'incarnation d'une œuvre d'art ne soit pas ce qui en fait un individu unique (un objet idéal peut être incarné à deux endroits à la fois) ${ }^{11}$; il y a là un type original de dépendance ontologique qui tolère l'« ubiquité», comme dit Valéry (l'existence d'un objet est en ce cas compatible avec l'existence simultanée d'une multiplicité de ces objets dont l'existence d'un seul (quel conque) lui est nécessaire ; en d'autres termes, si un objet detype $A$ existe, alors nécessairement un objet quelconque de type $B$ existe, mais il se peut que plusieurs $B$ existent en même temps). La notion d'idéalité liée semble alors contradictoire, puisque la dépendance ubique (caractéristique des idéalités) semble impliquer la dépendance non rigide (qui ne vaut que pour les idéalités libres).

Dans un autre genre, on peut vouloir distinguer une dépendance initiale et une dépendance continuée, selon que les termes de la relation de dépendance se succèdent nécessairement ou sont nécessairement contemporains. Par exemple, l'existence dans le temps d'un contrat de mariage dépend

10. Cette dimension temporelle est incontournable pour la plupart des ontologies du monde culturel puisqu'elle permet d'extraire les objets culturels du domaine des idéalités strictes. Cette dimension est soulignée, par exemple, par Roman Ingarden (L'đFuvre d'art littéraire), et particulièrement insistante lorsqu'il s'agit de proposer une ontologie du droit, comme chez R einach ( $D$ ie apriorischen $G$ rundlagen der bürgerlichen R echts), $M$ acCormick et Weinberger (An Institutional Theory of Law) ou Gardies (L'E rreur de H ume).

11. Ici, nous identifions, pour un objet idéal, le fait d'être incarné spatio-temporellement et le fait d'exister. Cela implique qu'un inventeur peut inventer un objet qui n'existe pas, si les seules traces de cette invention sont, par exemple, le récit d'une méthode de construction ou un schéma, ou même une image mentale. Cette conséquence ne nous paraît pas rédhibitoire, si l'on remarque que le verbe « inventer » a les mêmes propriétés que le verbe « chasser » analysé par Q uine : on peut inventer une machine impossible comme on peut chasser des licornes. 
initialement de certains actes de langage, mais de manière continuée de l'existence de chacun des époux.

Enfin, la notion de « self-dépendance » établie par Simons, reprise par Johansson dans ses I nvestigations ontologiques, dit qu'un objet nepeut exister en étant le seul spécimen de son espèce. Cette notion promet d'être importante dans le domaine social.

\section{Conclusion}

Cequ'il me paraît important de souligner, c'est que les différentes options qui s'offrent à celui qui veut traiter philosophiquement des objets culturels sont indépendantes par rapport à I'alternative réalisme/idéalisme. En effet, Stein et le second $\mathrm{H}$ usserl s'opposent sur la question du réal isme, mais s'accordent sur la stratégie d'une théorie des objets culturels. D'un autre côté, Carnap et H usserl partagent un phénoménalisme qu'on peut interpréter comme idéaliste, mais abordent les objets culturels selon des stratégies irréconciliables. Enfin, on se rappelle que l'alternative réalisme/idéalisme n'a même aucun sens pour Carnap.

L'alternative pertinente ici est plutôt celle qui existe entre l'approche extensionnaliste de Carnap et l'approche non extensionnaliste de H usserl. L'approche de Carnap n'entend pas condamner à l'inexistence les objets culturels du sens commun ou du juriste mais fait simplement remarquer que, dans un contexte scientifique, nous pouvons faire comme si deux objets différents étaient identiques. O n peut simplement, en retour, faire remarquer avec Goodman et Q uine que, pour tout programme réductionniste, qu'il soit scientifique ou philosophique, l'identité extensionnelle est un critère trop exigeant. Q uant à l'approche de $\mathrm{H}$ usserl, elle ne se résigne pas à substituer aux objets culturels des équivalents coextensifs, mais entend décrire les objets culturels tels qu'ils sont pensés et en tant que le monde actuel n'épuise pas toutes leurs ressources. C'est la stratégie que prône notamment J eanLouis Gardies, en substituant avantageusement les outils sémantiques au style phénoménologique. II faut néanmoins reconnaître que la stratégie réductionniste invite souvent à l'élimination ontologique et la stratégie «phénoménologique», dans une certaine tradition, au réalisme. On peut s'efforcer de n'y voir qu'une coïncidence.

Quoi qu'il en soit, cette conclusion aura au moins servi de prétexte pour rendre hommage à J ean-L ouis Gardies qui, en nous donnant à penser en compagnie de Reinach, de H usserl ou d'auteurs polonais, fut, en France, un ambassadeur dechoix pour la philosophie d'Europe centrale. Letextequi suit est I'ultime paragraphe de son avant-dernier livre, L es Fondements sémantiques du discours naturel. II y expose sa position sur les rapports entre I'ontologie et la sémantique. II ne faut pas s'étonner de ce que le terme « sémantique » n'apparaisse pas dans cet extrait, puisque tout l'effort de Gardies aura été de présenter comme équival ents les termes suivants : sémantique, phénoménologie et gnoséologie. Gardies écrit : 
Ainsi, si les considérations d'ordre gnoséologique qui ont été les nôtres ne sont sans doute pas susceptibles de s'adapter à n'importe quelle ontologie, il n'en reste pas moins qu'elles peuvent certainement s'adapter à des ontologies très différentes. N otre objet n'était nullement ici d'en sélectionner une ou d'en proposer une nouvelle, d'autant que nous aurions dû alors à cet effet quitter le terrain de la stricte analyse phénoménologique. Q u'il nous suffise de souligner que les seules ontologies envisageables sont celles dont l'agencement soit suffisant pour qu'il puisse, notamment, aujourd'hui s'y trouver que tel contenu propositionnel soit (ou ne soit pas) possible ou nécessaire, que le passé et le futur reçoivent leur place, qu'il y ait des ruptures et des changements, qu'il y ait aussi des actes, et non pas seulement des états, que tel individu s'engage (ou ne s'engage pas) envers tel autre, qu'il ait (ou n'ait pas) tel droit, telle obligation, telle connaissance ou telle illusion. ${ }^{12}$

\section{Bibliographie}

Carnap, R., Der Logische Aufbau der Welt, Berlin, Weltkreis, 1928 (traduction anglaise: The Logical Structure of the World, Londres, Routledge and Kegan Paul, 1967)

Gardies, J. -L., L'E rreur de H ume, Paris, PUF, 1987

- - , Les Fondements sémantiques du discours naturel, Paris, Vrin, 1994

Goodman, N., The Structure of A ppearance, Cambridge (M ass.), H arvard University Press, $3^{\mathrm{ème}}$ édition, Dordrecht, D. Reidel, 1977

H usserl, E., Expérience et J ugement, Paris, PUF, 1970

- - I Iées directrices pour une phénoménologie et une philosophie phénoménologique pures, livre second: Recherches phénoménologiques pour la constitution, Paris, PUF, 1982

Ingarden, R., L' Fuvre d'art littéraire, Lausanne, L'Âge d'H omme, 1983

Johansson, I., O ntological Investigations. An Inquiry into the Categories of

$\mathrm{N}$ ature, $\mathrm{M}$ an and Society, Londres, R outledge, 1989

M acCormick, N., Weinberger, O., An Institutional Theory of Law, Dordrecht, Kluwer, 1986

N eurath, O., «Empirical Sociology » (1931) in Empiricism and Sociology, Dordrecht, D. Reidel

Reinach, A., D ie apriorischen G rundlagen der bürgerlichen Rechts (1913), édité dans Sämtliche Werke, M ünchen $\mathrm{H}$ amden Wien, Philosophia Verlag, 1989

Simons, P., Parts: A Study in O ntology, Oxford, Oxford University Press, 1987

Stein, E., De l'État, Paris, Les Éditions du Cerf, 1989 